\title{
Prevalence of Physical Problems Detected by the Distress Thermometer and Problem List in Patients With Myeloproliferative Disorders
}

\author{
Daniel C. McFarland, DO; $;$ Kelly M. Shaffer, PhD; Heather Polizzi, RNc; John Mascarenhas, MD; \\ Marina Kremyanskaya, $\mathrm{MD}^{c}$; Jimmie Holland, $\mathrm{MD}^{\mathrm{b}}$; and Ronald Hoffman, MDc
}

\begin{abstract}
Background: Patients with myeloproliferative neoplasms (MPNs) can have a severe physical symptom burden over an extended disease trajectory that contributes to decreased quality of life. Few studies, however, have characterized which patients most frequently consider physical symptoms a problem. This study describes the physical symptoms of patients with MPNs and the relationship of these symptoms to patient characteristics. Methods: Patients with MPNs ( $\mathrm{N}=117)$ completed questionnaires in a dedicated academic medical center MPN clinic. Patients reported demographics (age, race/ethnicity, sex, marital status, employment status), disease characteristics (MPN type, time with MPN), and whether they were bothered by any of 22 variables in the "Physical Problems" list in the Distress Thermometer and Problem List (DT\&PL). Results: The median number of physical problems endorsed by patients was 2 (median, 2.26; SD, 3.18), with a range from 0 to 20. Two-fifths endorsed no physical problems, one-fifth endorsed 1 problem, and two-fifths endorsed $\geq 2$ problems, with fatigue (35.5\%), sleep $(27.1 \%)$, pain $(21.5 \%)$, dry skin/pruritus $(18.7 \%)$, and memory/concentration (16.8\%) being the most commonly reported. Non-Caucasian participants reported more problems with sleep $(P=.050)$, pain $(P=.016)$, and tingling $(P=.026)$. Patients with polycythemia vera (PV) reported more issues with tingling $(P=.046)$ and sexual problems $(P=.032)$. Conclusions: Patients with MPNs are more likely to report physical symptom bother than to report no bother with multiple physical problems on the DT\&PL. Patients of minority race/ethnicity and those with $\mathrm{PV}$, however, showed heightened prevalence of physical problems-characteristics which may be used to triage patients for more intensive symptom management.
\end{abstract}

Myeloproliferative neoplasms (MPNs) are a unique form of hematologic malignancy that are characterized by an elevated rate of physical symptom burden along a progressive and chronic disease trajectory. ${ }^{1}$ Patients with MPNs often report high physical symptom burden that has been shown to adversely affect their quality of life and psychological outcomes. ${ }^{2,3}$ Although it is known that patients with MPNs report frequent fatigue, early satiety, or pruritus depending on their MPN type and severity, little research has been performed to identify which physical problems are most commonly reported

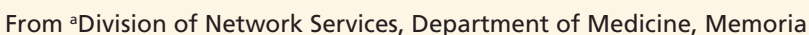
Sloan Kettering Cancer Center, West Harrison, New York; 'bepartment of Psychiatry and Behavioral Sciences, Memorial Sloan Kettering Cancer Center, New York, New York; and 'Division of Hematology/Oncology, Tisch Cancer Institute, Icahn School of Medicine at Mount Sinai Hospital, New York, New York.

Submitted May 6, 2017; accepted for publication August 2, 2017.
}

among patients with MPNs using the Distress Thermometer and Problem List (DT\&PL). The DT\&PL is endorsed by NCCN for the identification of distress in patients with cancer as a practical means to triage patients to appropriate resources, ${ }^{4}$ and it offers convenience and proven acceptability in busy oncology clinics. ${ }^{5}$ The accompanying Problem List provides a comprehensive reference of common physical symptoms experienced by patients with cancer. A better understanding of patients for whom symptoms are bothersome will be critical in order to target those patients most at risk for adverse

The authors have disclosed that they have no financial interests, arrangements, affiliations, or commercial interests with the manufacturers of any products discussed in this article or their competitors.

Correspondence: Daniel C. McFarland, DO, Division of Network Services, Department of Medicine, Memorial Sloan Kettering Cancer Center, 500 Westchester Avenue, West Harrison, NY 10604.

E-mail: danielcurtismcfarland@gmail.com 
outcomes related to physical problems for advanced symptom management interventions. In addition, physician reporting of symptoms has been shown to be less reliable than patient-reported symptoms. ${ }^{6}$ As such, this study is the first to characterize patientreported physical problems using the DT\&PL according to demographic and medical characteristics among patients with MPNs.

MPNs are represented by essential thrombocythemia (ET), polycythemia vera (PV), and myelofibrosis (MF), which all display variable but significant symptom burden. Across subtypes, patients with MPNs experience a unique constellation of physical symptoms, such as pruritus, night sweats, bone and splenic pain, fatigue, and fevers. Symptom burden can be similar in severity to the experience of patients with metastatic cancer or acute myeloid leukemia, but with a much longer period of overall survival, ${ }^{7}$ although symptom profiles and disease trajectories exist on a severity spectrum.

Physical symptom burden perceived by the individual is captured by the "Physical Problems" category of the DT\&PL, a helpful tool that is the most accepted and frequently implemented measure of distress used internationally $y^{8}$ and meets national distress screening mandates. ${ }^{8,9}$ The Problem List accompaniment includes a list of 22 physical symptoms and asks patients to indicate whether these have been a problem over the past week (yes/no). Capturing patients' subjective report of their physical symptom burden is important, given that doctors frequently underestimate patients' bother from physical symptoms, which may lead to inappropriate treatments and delayed referrals to palliative care. ${ }^{10,11}$ Patient self-reporting of symptom burden is strongly associated with their quality of life. ${ }^{12}$ Ours is the first study to use the DT\&PL as a patient-reported outcome (PRO) to characterize subjective physical symptom burden among a large, diverse sample of patients with MPNs.

Our study fills an important gap in the current literature, namely that there is little known regarding the physical problems on the DT\&PL or their associations with patient demographics and disease characteristics among those with MPNs. First, we characterize physical symptom burden self-reported by patients with MPNs. Next, we examine associations between endorsing the most frequent physical symptom burdens and patient characteristics.

\section{Methods}

The Mount Sinai Hospital Institutional Review Board approved this study in July 2014. Surveys were collected from participants from May to October 2015. All participants provided written informed consent to participate.

\section{Participants}

Men and women with documented MPNs were screened based on inclusion criteria consisting of a confirmed tissue diagnosis of an MPN, as reported by the treating physician. Patients who identified another cancer diagnosis were excluded from the study. Recruitment occurred over 4 months in a dedicated MPN clinic. New and established patients were recruited to participate in the survey.

\section{Procedure}

Participants were asked to participate by either a clinic receptionist or treating staff member (ie, nurse practitioner, hematologist) and were told that the survey was anonymous, was part of a research initiative, and that it did not relate to their ongoing care. Available psychological services were listed in the survey and patients were asked to bring up any concerns with clinic staff. A board certified psychiatrist oversaw the study and was available for consultation. Participants completed surveys while waiting in the clinic before or after their appointments and returned them directly to clinic staff.

\section{Measures}

Patient Demographics and Medical Characteristics: Patients reported demographic information, including age, race/ethnicity, sex, and marital and employment status, as well as medical information, including disease type (ET, PV, MF, "other MPN" [eg, hypereosinophilic syndrome, PDGFRA-positive MPN, systemic mastocytosis, or chronic neutrophilic leukemia]) and length of time with disease $(<1$ year, 1 to $<3$ years, 3 to $<5$ years, 5 to $<10$ years, $\geq 10$ years).

Physical Problems: Patients endorsed whether a physical symptom had been a problem for them over the past week using the "Physical Problems" list within the DT\&PL, which contains 22 separate items (Table 1). The DT\&PL has been used widely by cancer institutions to meet the Commission on 
Physical Problem Detection in MPNs

\section{Table 1. Frequency of Physical Problems Endorsed}

\begin{tabular}{|lcc|}
\hline Physical Problem List Variables & N & $\%$ \\
\hline Fatigue & 38 & 35.5 \\
\hline Sleep & 29 & 27.1 \\
\hline Pain & 23 & 21.5 \\
\hline Skin dry/itchy & 20 & 18.7 \\
\hline Tingling in hands/feet & 19 & 17.8 \\
\hline Memory/Concentration & 18 & 16.8 \\
\hline Feeling swollen & 12 & 11.2 \\
\hline Breathing & 11 & 10.3 \\
\hline Sexual & 11 & 10.3 \\
\hline Mouth sores & 10 & 9.3 \\
\hline Appearance & 9 & 8.4 \\
\hline Constipation & 9 & 8.4 \\
\hline Diarrhea & 9 & 8.4 \\
\hline Getting around & 9 & 8.4 \\
\hline Nose dry/congested & 9 & 8.4 \\
\hline Eating & 7 & 6.5 \\
\hline Indigestion & 6 & 5.6 \\
\hline Nausea & 2 & 4.7 \\
\hline Change in urination & 5 & 3.7 \\
\hline Bathing/Dressing & 4 & 1.9 \\
\hline Fevers & 2 & 1.9 \\
\hline Substance abuse & 2 & 1.9 \\
\hline & 2 & \\
\hline
\end{tabular}

Cancer distress-screening mandate for accreditation in 2015. ${ }^{9}$

\section{Statistical Analysis}

The primary outcome of this study was to determine the prevalence of the 22 physical problem variables listed in the DT\&PL. Associations with demographics (age, race/ethnicity, sex, and marital and employment status) and disease characteristics (MPN type, time with MPN) were examined if at least $10 \%$ of patients endorsed that particular physical symptom (to ensure adequate power). Independent $t$-tests were used to assess the bivariate associations between patient age and endorsement of physical problems. Chisquare tests were used to assess the bivariate associations between categorical patient characteristics and endorsement of physical problems. For significant chi-square tests with disease characteristic variables, follow-up pairwise comparisons were conducted to compare proportions of physical problem endorsement among variable levels. Keppel's modified Bonferroni correction was used to control for type I er- ror at the 0.05 level across follow-up comparisons. ${ }^{13}$ Statistical procedures were performed using SPSS version 22 (IBM Corp., Armonk, NY) and statistical tests were 2 -tailed with a $5 \%$ significance level.

\section{Results}

Characteristics of the 117 patients with confirmed MPN who completed the survey (78\% response rate) are listed in Table 2. The average patient age was 57.7 years $(\mathrm{SD}, 14.8)$, and 69 respondents $(60.5 \%)$ were female. A total of 34 respondents $(31.2 \%)$ had PV, 31 (28.4\%) had ET, 31 (28.4\%) had MF, and $13(11.9 \%)$ had another type of MPN. The sample comprised approximately half of patients with an MPN for $<5$ years versus those with the disease for $\geq 5$ years. Most participants were Caucasian (76.1\%),

Table 2. Patient Demographics and Characteristics

\begin{tabular}{|c|c|c|c|}
\hline & & $\begin{array}{c}n^{\mathrm{a}} \\
(\mathrm{N}=117)\end{array}$ & $\%$ \\
\hline Mean age, y (SD) & $57.7(14.8)$ & & \\
\hline \multicolumn{4}{|l|}{ Race/Ethnicity } \\
\hline Caucasian & & 86 & 76.1 \\
\hline Non-Caucasian & & 27 & 23.9 \\
\hline \multicolumn{4}{|l|}{ Sex } \\
\hline Female & & 69 & 60.5 \\
\hline Male & & 45 & 39.5 \\
\hline \multicolumn{4}{|l|}{ Married/Partnered } \\
\hline Yes & & 73 & 65.2 \\
\hline No & & 39 & 34.8 \\
\hline \multicolumn{4}{|l|}{ Employed } \\
\hline Yes & & 63 & 55.8 \\
\hline No & & 50 & 44.2 \\
\hline \multicolumn{4}{|l|}{ Disease type } \\
\hline PV & & 34 & 31.2 \\
\hline ET & & 31 & 28.4 \\
\hline MF & & 31 & 28.4 \\
\hline Other & & 13 & 11.9 \\
\hline \multicolumn{4}{|l|}{ Time with MPN, y } \\
\hline$<1$ & & 8 & 7.5 \\
\hline 1 to $<3$ & & 21 & 19.8 \\
\hline 3 to $<5$ & & 25 & 23.6 \\
\hline 5 to $<10$ & & 21 & 19.8 \\
\hline$\geq 10$ & & 31 & 29.2 \\
\hline
\end{tabular}

Where sum $>117$, patients did not report data.

Abbreviations: ET, essential thrombocythemia; MF, myelofibrosis; MPN, myeloproliferative neoplasm; PV, polycythemia vera. 
partnered or married (65.2\%), and working (55.8\%). Other races/ethnicities included in the non-Caucasian population were Latin/Hispanic $(n=11 ; 10.1 \%)$, black $(n=8 ; 7.3 \%)$, Asian $(n=3 ; 2.8 \%)$, and other $(n=5 ; 4.6 \%)$.

The median number of physical problem variables listed in the DT\&PL endorsed by patients was $2($ mean $=2.26 ; \mathrm{SD}=3.18)$ with a range from 0 to 20 (see Table 1). Two-fifths of the patients endorsed no physical problems, one-fifth endorsed 1 problem, and two-fifths endorsed $\geq 2$ problems. The 5 most commonly reported physical problems were fatigue (35.5\%), sleep $(27.1 \%)$, pain $(21.5 \%)$, skin dry/ itchy (ie, pruritus; $18.7 \%$ ), and tingling in hands/feet (17.8\%). Problems with bathing/dressing (1.9\%), fevers $(1.9 \%)$, and substance abuse $(1.9 \%)$ were not commonly reported.

Results of bivariate comparison tests examining the associations between patient characteristics and endorsement of physical problems are listed in supplemental eTable 1 (available with this article at INCCN.org). Non-Caucasian participants reported more problems with sleep $(P=.050)$, pain $(P=.016)$, and tingling $(P=.026)$. For non-Caucasian versus Caucasian participants, the probability of reporting a problem was 1.8 times higher for sleep $(0.458 \mathrm{vs}$ $0.261), 2.3$ times higher for pain $(0.435$ vs 0.186$)$, and 2.6 times higher for tingling (0.296 vs 0.116 ). Patients who were married/partnered had less difficulty with fatigue $(P=.008)$. The probability of reporting a problem with fatigue was 2 times higher for unmarried participants versus married/partnered ones (0.576 vs 0.293 ).

Patients differed in reports of tingling $(P=.046)$ and sexual problems $(P=.032)$ across MPN disease type. For tingling, follow-up planned comparisons using Keppel's modified Bonferroni correction $\left(\alpha_{p c}=0.044\right)$ revealed that the only significant pairwise difference was between ET and other MPN $\left(\chi^{2}[1, n=45]=8.809 ; P=.003\right)$. The probability of reporting a problem with tingling was 11 times higher for those with MPNs versus ET (0.357 vs 0.032). For sexual problems, follow-up planned comparisons using Keppel's modified Bonferroni correction $\left(\alpha_{\mathrm{pc}}=0.044\right)$ revealed that the only significant pairwise difference was between PV and ET $\left(\chi^{2}[1\right.$, $\mathrm{n}=54]=6.533 ; P=.011)$. The probability of reporting sexual problems was 8 times higher for those with PV than those with ET (0.296 vs 0.037). Age, sex, em- ployment status, and time with MPN were not significantly associated with endorsing variables in the "Physical Problems" list in the DT\&PL, although trends $(P<.10)$ emerged that warrant further study.

\section{Discussion}

This study is the first to characterize patient-reported physical symptom burden (ie, PROs) using the DT\&PL among a large, diverse sample of patients with MPNs. The DT\&PL is a short and convenient measure that is already most commonly used to screen for distress internationally and may provide a convenient way to also screen physical symptom burden in patients with MPNs. Equal proportions of the sample reported no physical problems and $\geq 2$ physical problems, with fatigue and sleep disturbance reported by more than one-quarter of the sample. Several patient demographic and medical characteristics were associated with likelihood of endorsing a physical problem. Findings suggest that particular patient characteristics may be used to target symptom management interventions to those at highest risk for adverse outcomes related to these problems.

The order of symptom occurrence in the current study fits with prior studies of MPNs where the most commonly endorsed physical symptom among patients was consistently fatigue. ${ }^{7,14}$ However, the prevalence of endorsed symptoms is lower than in previous studies. ${ }^{2,7}$ This difference may be explained by the dichotomous answer types (yes/no) of the DT\&PL versus a 0 to 10 analogue scale that may provide greater sensitivity. Another explanation may be due to the wording of the DT\&PL, which asks whether a patient has "been bothered by" a physical symptom, rather than simply "experienced" the symptom. The DT\&PL may select for greater symptom severity. The potential advantage of using the DT\&PL in patients with MPNs to monitor for physical symptom is its brevity and acceptability among general oncology clinics. ${ }^{5}$ Clearly, validated measures for symptom burden in MPN may be more sensitive and specific, but our analysis provides an opportunity to understand MPN symptomatology with this more general tool that is in use in many clinics.

Fatigue, the most commonly reported symptom, may be mediated by increased cytokine release in patients with MPNs, because this is a clonal disease leading to excessive production of protein me- 
Physical Problem Detection in MPNs

diators. ${ }^{15,16}$ Interestingly, having a spouse or partner appeared to be protective against fatigue. Romantic partners can exert a positive effect on one's lifestyle that may lessen fatigue, such as promoting healthy lifestyle behaviors, ${ }^{17}$ consistent social routines, ${ }^{18}$ and behavioral activation during the day. ${ }^{18,19}$

Non-Caucasian patients with MPNs more commonly reported physical issues of tingling (eg, peripheral neuropathy), sleep, and pain compared with Caucasian patients. This may represent undertreatment of these symptoms among non-Caucasian patients, an ethnically distinct symptom producing mechanism inherent to MPNs, or cultural differences in symptom reporting. For example, Asian-variant MPNs are increasingly reported. ${ }^{20,21}$ However, reporting of more problems with tingling, sleep, and pain among nonCaucasians is consistent with other studies that show greater symptom burden among African American and Hispanic ethnicities and undertreatment of those symptoms in various subsets of cancer. ${ }^{22,23}$ Additionally, there may be more barriers to managing cancerrelated pain in the advanced cancer setting consistent with known disparities in pain management, which may also be seen in the MPN setting. ${ }^{24}$

Patients with MPN diagnoses other than the 3 major subtypes reported more neuropathy than those with ET, whereas patients with PV reported more sexual problems than those with ET. This finding is consistent with the underlying vascular pathophysiology that leads to sexual impotence in men. ${ }^{25}$ Therefore, clinicians should be particularly vigilant of symptoms of neuropathy and sexual dysfunction reported on the DT\&PL by patients with PV. These symptoms are known to be common in patients with $\mathrm{PV}^{26,27}$ and are also prevalent in patients with MF. ${ }^{28}$ Pruritus was not found to be exclusively associated with PV, because it may be present in other MPNs and may have been mitigated by length of time with MPN, adequate treatments, and/or effective coping. Further research to test these hypotheses is warranted. Also, a further analysis of the specific diseases in the "other" group may be helpful because this group experienced greater neuropathy.

The primary limitation to this study is the use of a nonvalidated tool (the physical problems list from the DT\&PL) specifically for measuring physical symptom burden, as opposed to measures such as the MPN Symptom Assessment Form (MPN-SAF) ${ }^{7}$ or the Functional Assessment of Cancer TherapyAnemia (FACT-An), ${ }^{29}$ which have been validated as PRO measures and capture frequency and severity. ${ }^{7,29}$ Additionally, the number of participants is low relative to the MPN-SAF and its validation studies, which complicates the interpretation of our findings. However, the DT\&PL showed the presence of physical symptom burden albeit at a lower prevalence than prior studies that used longer quality-of-life measures. ${ }^{2,7}$ This suggests the utility of using the DT\&PL has confirmed clinic acceptability and is rapidly completed by patients, ${ }^{5,8}$ although further study should test this hypothesis and establish validity in the MPN population. As data are crosssectional, future research should seek to examine associations between patient characteristics and the development of physical symptom burden from MPN longitudinally. Furthermore, as several of the commonly reported symptoms (eg, fatigue, sleep, pain, memory/concentration problems) may also be related to psychological symptoms elevated in MPNs, future research should examine the interrelations between MPNs' physical and psychological symptom burdens. ${ }^{3}$

\section{Conclusions}

This study provides evidence for an association between certain physical symptom problems and MPN disease type and patient demographics using the DT\&PL. Two-fifths of patients reported $\geq 2$ physical symptoms that were problematic and thus not adequately controlled. Patients who were non-Caucasian and unmarried were more likely to report certain problems relative to Caucasian and married/partnered patients, whereas those with ET were less likely to report certain problems relative to those with PV or another MPN than the 3 major subtypes. The DT\&PL, widely implemented as a distress-screening instrument, may also be helpful in delineating problematic physical symptomatology in patients with MPNs. The development of targeted, effective symptom management strategies for patients with MPN at highest risk for adverse outcomes from physical problems associated with the disease and treatment will be critical to sustaining the quality of life among this vulnerable population of chronically ill patients. 
McFarland et al

\section{References}

1. Mesa R, Miller CB, Thyne M, et al. Myeloproliferative neoplasms (MPNs) have a significant impact on patients' overall health and productivity: the MPN Landmark survey. BMC Cancer 2016;16:167.

2. Mesa RA, Niblack J, Wadleigh M, et al. The burden of fatigue and quality of life in myeloproliferative disorders (MPDs): an international Internetbased survey of 1179 MPD patients. Cancer 2007;109:68-76.

3. McFarland DC, Polizzi H, Mascarenhas J, et al. Psychological symptoms among patients with BCR-ABL-negative myeloproliferative neoplasms. J Natl Compr Canc Netw 2016;14:1563-1570.

4. Holland JC, Deshields TL, Andersen B, et al. NCCN Clinical Practice Guidelines in Oncology: Distress Management. Version 3.2015. Accessed December 22, 2015. To view the most recent version of these guidelines, visit NCCN.org.

5. Mitchell AJ. Short screening tools for cancer-related distress: a review and diagnostic validity meta-analysis. J Natl Compr Canc Netw 2010;8:487494.

6. Mesa RA, Miller CB, Thyne M, et al. Differences in treatment goals and perception of symptom burden between patients with myeloproliferative neoplasms (MPNs) and hematologists/oncologists in the United States: findings from the MPN Landmark survey. Cancer 2017;123:449-458.

7. Scherber R, Dueck AC, Johansson P, et al. The Myeloproliferative Neoplasm Symptom Assessment Form (MPN-SAF): international prospective validation and reliability trial in 402 patients. Blood 2011;118:401-408.

8. Donovan KA, Grassi L, McGinty HL, Jacobsen PB. Validation of the distress thermometer worldwide: state of the science. Psychooncology 2014;23:241-250.

9. Pirl WF, Fann JR, Greer JA, et al. Recommendations for the implementation of distress screening programs in cancer centers: report from the American Psychosocial Oncology Society (APOS), Association of Oncology Social Work (AOSW), and Oncology Nursing Society (ONS) joint task force. Cancer 2014;120:2946-2954.

10. Tishelman C, Petersson LM, Degner LF, Sprangers MA. Symptom prevalence, intensity, and distress in patients with inoperable lung cancer in relation to time of death. J Clin Oncol 2007;25:5381-5389.

11. Portenoy RK, Thaler HT, Kornblith AB, et al. Symptom prevalence, characteristics and distress in a cancer population. Qual Life Res 1994;3:183-189.

12. Osoba D. Translating the science of patient-reported outcomes assessment into clinical practice. J Natl Cancer Inst Monogr 2007:5-11.

13. Keppel G. Design and Analysis: A Researcher's Handbook, 3rd ed. Englewood Clifts, NY: Prentice-Hall, Inc; 1991.
14. Scherber RM, Kosiorek HE, Senyak Z, et al. Comprehensively understanding fatigue in patients with myeloproliferative neoplasms. Cancer 2016;122:477-485.

15. Geyer HL, Dueck AC, Scherber RM, Mesa RA. Impact of inflammation on myeloproliferative neoplasm symptom development. Mediators Inflamm 2015;2015:284706.

16. Mondet J, Hussein K, Mossuz P. Circulating cytokine levels as marker of inflammation in philadelphia negative myeloproliferative neoplasms: diagnostic and prognostic interest. Mediators Inflamm 2015;2015:670580.

17. Fuller T. Relationship status, health, and health behavior: an examination of cohabitators and commuters. Sociological Perspectives 2010;53:221245.

18. Carney CE, Edinger JD, Meyer B, et al. Daily activities and sleep quality in college students. Chronobiol Int 2006;23:623-637.

19. Monk TH, Petrie SR, Hayes AJ, Kupfer DJ. Regularity of daily life in relation to personality, age, gender, sleep quality and circadian rhythms. J Sleep Res 1994;3:196-205.

20. Gill H, Leung AY, Chan CC, et al. Clinicopathologic features and prognostic indicators in Chinese patients with myelofibrosis. Hematology 2016;21:10-18

21. $\mathrm{Xu} \mathrm{Z}$, Gale RP, Zhang $\mathrm{Y}$, et al. Unique features of primary myelofibrosis in Chinese. Blood 2012;119:2469-2473.

22. Morrow PK, Broxson AC, Munsell MF, et al. Effect of age and race on quality of life in young breast cancer survivors. Clin Breast Cancer 2014;14:e21-31.

23. Fu OS, Crew KD, Jacobson JS, et al. Ethnicity and persistent symptom burden in breast cancer survivors. J Cancer Surviv 2009;3:241-250.

24. Campbell CM, Edwards RR. Ethnic differences in pain and pain management. Pain Manag 2012;2:219-230.

25. Michiels JJ. Erythromelalgia and vascular complications in polycythemia vera. Semin Thromb Hemost 1997;23:441-454.

26. Yiannikas C, McLeod JG, Walsh JC. Peripheral neuropathy associated with polycythemia vera. Neurology 1983;33:139-143.

27. Bouomrani S, Farah A, Nefoussi M, et al. Erectile dysfunction in polycythemia vera [in French]. Presse Med 2012;41:546-548.

28. Geyer HL, Andreasson B, Kosiorek HE, et al. The role of sexuality symptoms in myeloproliferative neoplasm symptom burden and quality of life: an analysis by the MPN QOL International Study Group. Cancer 2016;122:1888-1896

29. Tefferi A, Hudgens S, Mesa R, et al. Use of the Functional Assessment of Cancer Therapy—anemia in persons with myeloproliferative neoplasmassociated myelofibrosis and anemia. Clin Ther 2014;36:560-566.

\section{See JNCCN.org for supplemental online content.}

